\title{
Measuring the muon content of air showers with IceTop
}

\author{
Javier G. Gonzalez for the IceCube Collaboration \\ University of Delaware
}

\begin{abstract}
IceTop, the surface component of the IceCube detector, has been used to measure the energy spectrum of cosmic ray primaries in the range between $1.58 \mathrm{PeV}$ and $1.26 \mathrm{EeV}$. It can also be used to study the low energy muons in air showers by looking at large distances $(>300 \mathrm{~m})$ from the shower axis. We will show the muon lateral distribution function at large lateral distances as measured with IceTop and discuss the implications of this measurement. We will also discuss the prospects for low energy muon studies with IceTop.
\end{abstract}

It is well known that the muon content of an air shower, together with a measure of its electromagnetic component, can be used to estimate the energy and mass of its primary [1]. The main issue with the use of the muon content as an estimate of primary mass is the possible systematic differences between simulated and real air showers, arising from the lack of knowledge of high energy hadronic interactions. An excess number of muons has been reported by the HiRes-MIA and the Pierre Auger collaborations [2,3]. Understanding the systematic differences in air shower muon content between simulations and data is one of the pressing issues in the physics of very high energy cosmic rays.

The IceTop detector is sensitive to the low-energy $(\mathrm{E} \gtrsim 200 \mathrm{MeV})$ muon component of air showers. Generally speaking, at low zenith angle and close enough to the air shower axis, the signal from muons is overwhelmed by the signal from the electromagnetic (EM) component of the air shower (electrons, positrons and gamma-rays). This holds true in the zenith angle and lateral distance ranges that have been used in the cosmic ray spectrum determination with IceTop $\left(\theta<40^{\circ}, \mathrm{r} \lesssim\right.$ $\left.220 \cdot(E / P e V)^{1 / 4} \mathrm{~m}\right)$. We are interested in determining the average muon Lateral Distribution Function (LDF) at lateral distances where the signal from muons becomes significant. The lateral distance of any point is defined as the closest distance from the point to the shower axis.

The estimation of the muon lateral distribution hinges on being able to distinguish the signal produced by single muons from the signal produced by EM particles. IceTop detector stations accomplish this by virtue of their size. A typical electron leaves a signal proportional to its track length through the detector, which is on the order of $10 \mathrm{~cm}$. A typical muon crosses the detector, producing a track typically longer than $90 \mathrm{~cm}$. After describing the general features of IceTop signals in Sect. 1, we will describe a method for measuring the number of muons in Sect. 2. This method does not rely on counting muons, but it is a fit to the signal distributions within small bins of lateral distance, primary energy and zenith angle.

\section{General features of IceTop}

The IceCube detector consists of two major components. It can measure air showers on the surface with IceTop, high energy muon bundles with the in-ice detector, and both components in coincidence provided that the air shower axis goes through the in-ice detector. In what follows, we will consider the specific characteristics of IceTop that are relevant for measuring the low-energy muon component of air showers. A more detailed description of IceCube and IceTop has already been presented elsewhere [4].

IceTop is an air shower array consisting of 81 stations forming a triangular grid with a separation of $125 \mathrm{~m}$ in its completed configuration. The results presented here were obtained with data collected between June 1st 2010 and May 13th 2011, when IceTop consisted of 73 stations. It is located above the deep IceCube detector at the geographical South Pole, covering an area of roughly one square kilometer. Each station consists of two ice Cherenkov tanks separated by ten meters. Each tank contains two Digital Optical Modules (DOMs) with a 10 inch photomultiplier tube (PMT) and electronics for signal processing and readout. A discriminator trigger occurs when the voltage in one of the DOMs in a tank has passed the discriminator threshold. The total charge collected at the PMT's anode, after digitization and baseline subtraction, constitutes the tank's signal. The tanks register signals ranging from 0.2 to 1000 Vertical Equivalent Muons (VEM). A Hard Local Coincidence (HLC) occurs when there are discriminator triggers in two neighboring tanks within a time window of $1 \mu \mathrm{s}$. If there is a discriminator trigger but not an HLC, the result is a Soft Local Coincidence (SLC).

All analyses of IceTop data up to now have only considered signals where both tanks in a station pass the threshold, or HLC signals. In this contribution we also consider SLC signals, where the partner tank within the station did not have a discriminator trigger. SLC signals occur at large lateral distances, where the triggering probability is smallest. An example of the lateral

This is an Open Access article distributed under the terms of the Creative Commons Attribution License 4.0, which permits unrestricted use, distribution, and reproduction in any medium, provided the original work is properly cited. 


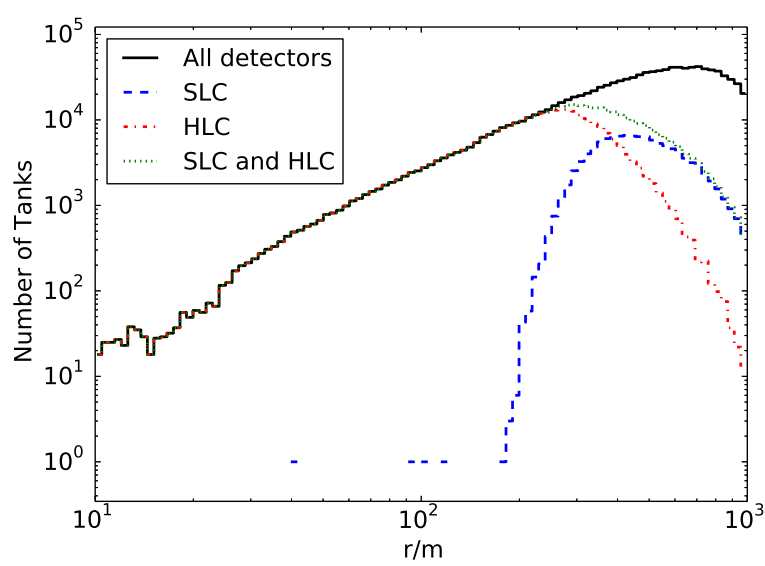

Figure 1. Lateral distribution of tanks in showers arriving with zenith angles less than $6^{\circ}$ and with energies between $10 \mathrm{PeV}$ and 12.6 PeV. The tanks are classified depending on whether they registered $S L C$ or $H L C$ signals (described in the text). The upper curve includes all tanks, including tanks that registered no signal during the event. In determining the muon content, we will only consider tanks at lateral distances larger than where the SLC and HLC lines cross.

distribution of SLC and HLC signals from experimental data is shown in Fig. 1.

The properties of the primary cosmic ray are reconstructed by fitting the measured signals with a Lateral Distribution Function (LDF) which includes an attenuation factor due to the snow cover on top of each tank. For a given primary energy and arrival direction, the observed lateral distribution of signals used in this reconstruction is shown in Fig. 2. The signal times are fitted with a function describing the shape of the shower front. The primary energy is given by the shower size $\mathrm{S}_{125}$, defined as the signal at a lateral distance of $125 \mathrm{~m}$. The resulting cosmic ray energy spectrum measured with IceCube/IceTop has been presented previously [5-7]. In the shower reconstruction just outlined, only HLC signals were considered.

The distinction between SLC and HLC signals provides a natural way to identify tanks where we expect to see a significant muon contribution. Generally speaking, we expect that signals at large lateral distances will be mostly due to muons, whereas the signals at short lateral distances will be mostly due to electrons and $\gamma$-rays. Instead of relying on simulations for selecting signals where the contribution from muons dominate, we will select tanks where SLC signals are most likely. Specifically, we will restrict ourselves to tanks beyond a lateral distance at which SLC signals amount to $50 \%$ of the total number of signals. The exact lateral distance at which this happens depends on the energy of the primary. The dependence on zenith angle is very weak. Therefore, we chose to use the value corresponding to $30^{\circ}$.

The statistical distribution of signals from EM particles will roughly mimic their energy distribution, with a mean signal that corresponds to a few tens of centimeters of track length inside the tank. On the other hand, the distribution of signals from muons is mainly determined by the geometry of the tank. The signal distributions produced by single muons are obtained using the Geant 4

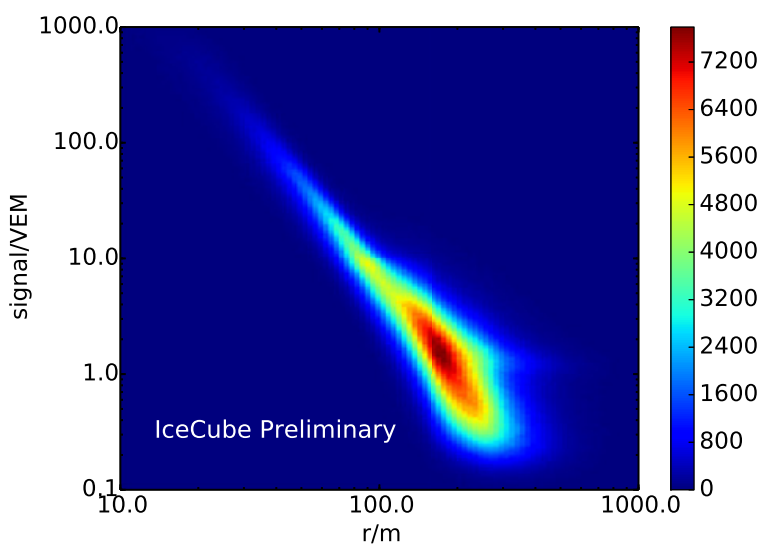

Figure 2. HLC signal distribution as a function of lateral distance for air showers with energies between 4 and $5 \mathrm{PeV}$, and zenith angle between $28^{\circ}$ and $32^{\circ}$. This distribution is a $2 \mathrm{~d}$ histogram that includes all HLC signals from all events with the given energy and arrival direction.

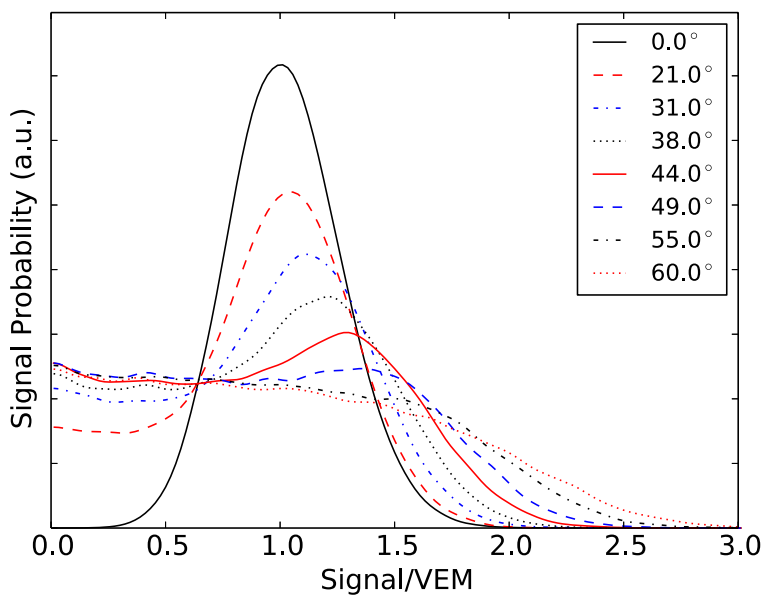

Figure 3. Signal distributions resulting from simulating the detector response for single muons arriving at fixed zenith angles from $0^{\circ}$ to $57^{\circ}$.

toolkit [8]. Example distributions at various incident angles are displayed in Fig. 3. The distribution is clearly not symmetric. The peak of the distribution corresponds to muons that enter through the top of the tank and exit through the bottom. By definition, the peak position for vertically through-going muons is one Vertical Equivalent Muon (VEM). For muons arriving at a zenith angle $\theta$, the peak is at $1 / \cos (\theta)$. The flat part at low signals corresponds to muons with a short track through the tank, what we call corner clipping muons. At large angles, few muons go through top and bottom. For an integer number of muons, the signal distribution is just the multiple auto-convolution of the single-particle distribution. An example of this is displayed in Fig. 4. The total signal distribution for an expected average number of muons $\left\langle N_{\mu}\right\rangle$ is given by a linear combination of the signal distributions for integer numbers of muons:

$$
p\left(s \mid\left\langle N_{\mu}\right\rangle\right)=\sum_{n=0}^{\infty} \frac{\left\langle N_{\mu}\right\rangle^{n}}{n !} e^{-\left\langle N_{\mu}\right\rangle} p\left(s \mid N_{\mu}=n\right) .
$$




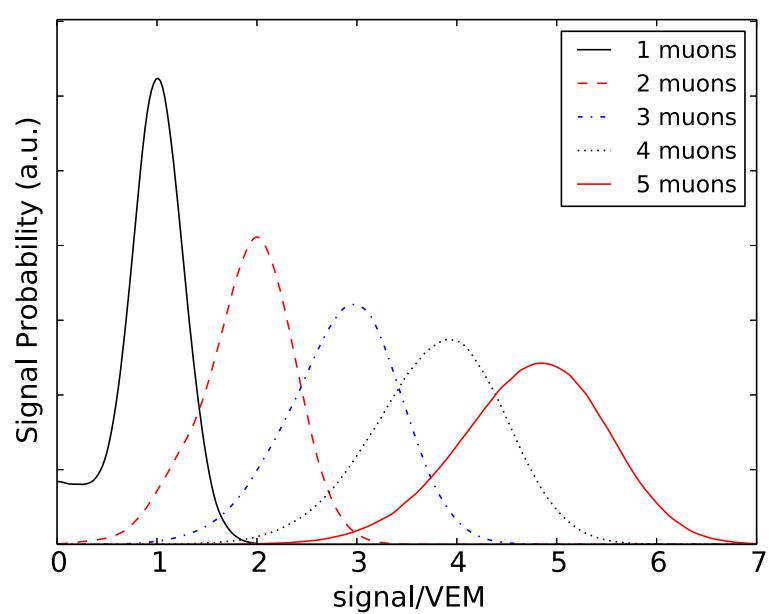

Figure 4. Signal distributions resulting from simulating the detector response for an integer number of muons arriving arriving at $10^{\circ}$.

\section{Determining the muon lateral distribution}

An example of the observed distribution of IceTop signals, corresponding to air showers with fixed energy and zenith angle, can be seen in Fig. 5, where we now included the SLC signals. At large distances, there are two distinct populations. One population is the continuation of the main distribution at smaller distances, which roughly follows a power law, where the electromagnetic component of the shower dominates. The other population, with signals around 1 VEM, is made up mostly of tanks hit by one or more muons. These two populations are clearly seen in Fig. 6, where we show the histograms of signals registered at selected fixed lateral distances.

The first population corresponds to tanks hit by no muons. The entire signal in these tanks is produced by electrons or $\gamma$-rays. We approximate this distribution by a power-law multiplied by a function that describes the trigger probability. The trigger probability can be described by a sigmoidal function of the logarithm of the charge, centered at $0.25 \mathrm{VEM}$ and with a width of 0.14 . Clearly, this approximation only works well at large lateral distances, where the mean expected signal is well below threshold and we thus look into the tail of the signal distribution. The second population, with a peak around $1 \mathrm{VEM} / \cos \theta$, can be described by the contributions of tanks hit by an integer number of muons. The charge distribution obtained from simulations was described in Sect. 1 and is given by Eq. (1). This distribution is smeared and shifted to account for a very small contribution from electrons, positrons, and $\gamma$-rays.

We determine the muon lateral distribution function by independently fitting the charge distributions at fixed energy, zenith, and lateral distance, like the distributions shown in Fig. 6. The result of the fit is the number of tanks hit by at least one muon. This, together with the total number of tanks located at that distance (the upper curve in Fig. 1), provides an estimate of the probability that a

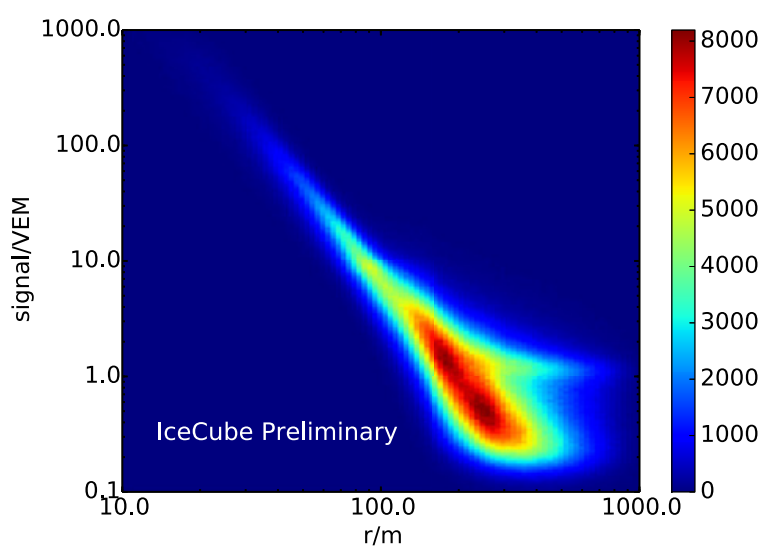

Figure 5. Signal distribution as a function of lateral distance for air showers with energies between 4 and $5 \mathrm{PeV}$, and zenith angle between $28^{\circ}$ and $32^{\circ}$. This distribution includes all signals (SLC and HLC). The vertical lines mark the distances at which the 1-d histograms in Fig. 6 were made.
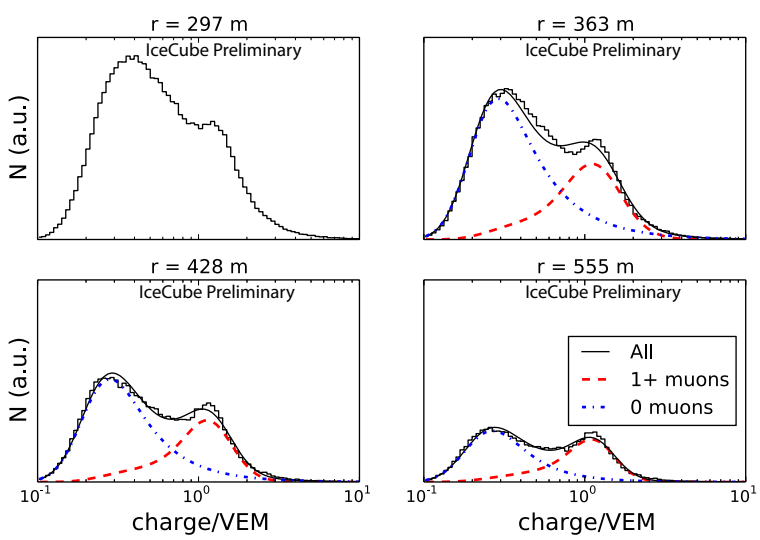

Figure 6. Vertical slices of the 2-d histogram in Fig. 5. Each histogram corresponds to a vertical line in Fig. 5. The fit lines are not shown at $297 \mathrm{~m}$ because at this point the assumption made of the shape of the EM signal, that the peak EM signal is below threshold, is not valid.

tank is hit by one or more muons, which leads to the mean number of muons $\left\langle N_{\mu}\right\rangle$ :

$$
p_{\mu h i t}=\frac{N_{\text {tanks withmuons }}}{N_{\text {all tanks }}}=1-e^{-\left\langle N_{\mu}\right\rangle} \text {. }
$$

The mean number of muons is divided by the crosssectional area of the tanks to yield the muon density at that location. In doing this, we assume that the direction of motion of all the muons coincide with the reconstructed air shower direction. The resulting muon density for each lateral distance and some of the energies considered, at two different zenith angles $\left(0^{\circ}\right.$ and $31^{\circ}$ from the vertical), are shown in Figs. 7 and 8. At this point we should remember that all the points in these figures are chosen so the number of SLC signals at any of the considered lateral distances are at least $50 \%$ of the number of signals at that lateral distance. The smaller and fainter markers correspond to tanks located at lateral distances in which SLC signals amount to less than $80 \%$ of all signals at that distance. Clearly, increasing this lateral distance cut enhances the muon's contribution to the signal, but at the 


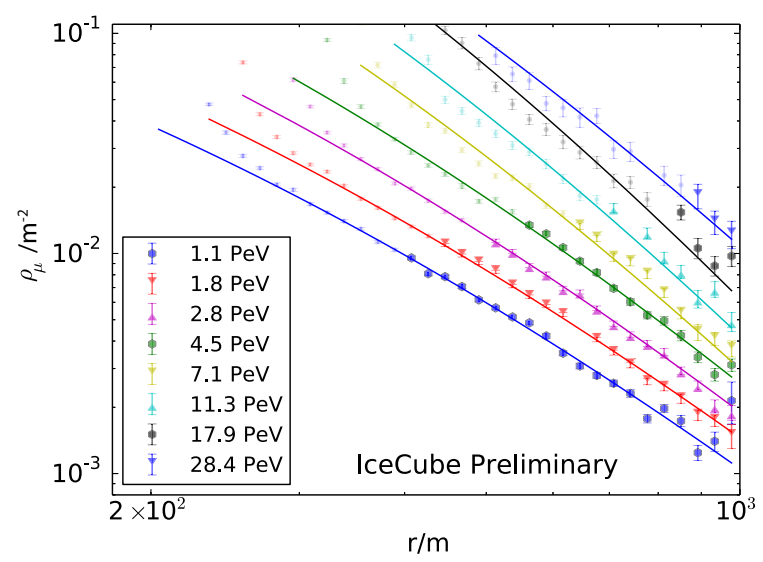

Figure 7. Reconstructed average lateral distribution of muons for air showers arriving at zenith angles of less than $6^{\circ}$ and selected $\mathrm{S}_{125}$ values.

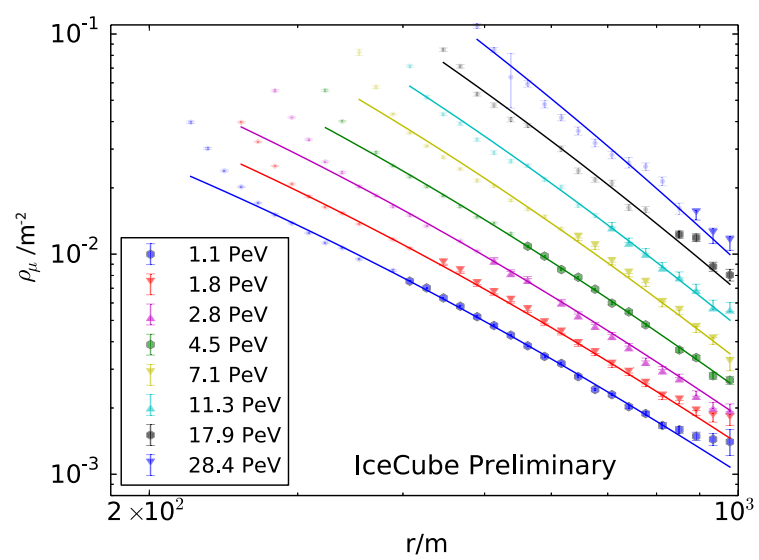

Figure 8. Reconstructed average lateral distribution of muons for air showers arriving at zenith angles between $28^{\circ}$ and $31^{\circ}$, and selected $S_{125}$ values.

same time limits the accuracy of the lateral distribution fit. The optimization of this cut remains under study.

Each LDF can be described by the following function [9]:

$$
\rho_{\mu}(r)=\rho_{\mu}\left(r_{0}\right)\left(\frac{r}{r_{0}}\right)^{-3 / 4}\left(\frac{320 \mathrm{~m}+r}{320 \mathrm{~m}+r_{0}}\right)^{-\gamma},
$$

which displays the same functional form as Greisen's function, with the first exponent of $r$ fixed to $-3 / 4$. The parameters that are fitted are $\gamma$ and $\rho_{\mu}\left(r_{0}\right)$, which represents the density of muons at a given lateral distance $r_{0}$. We have chosen 600 meters as the value for $r_{0}$. The choice of $r_{0}$ is arbitrary and is motivated by the fact that the signals shown in Figs. 7 and 8 are at lateral distances between 300 and 1000 meters. It is also convenient because this is the lateral distance at which previous experiments, notably Akeno and HiRes-MIA [2,10,11], reported their results. It must be noted that the optimum value for $r_{0}$ depends on energy and on the lateral distance cut. The two parameters, $\rho_{\mu}\left(r_{0}\right)$ and $\gamma$, potentially depend on energy and zenith angle. In the following section we will show how $\rho_{\mu}\left(r_{0}\right)$ depends on energy in the case of vertical air showers.

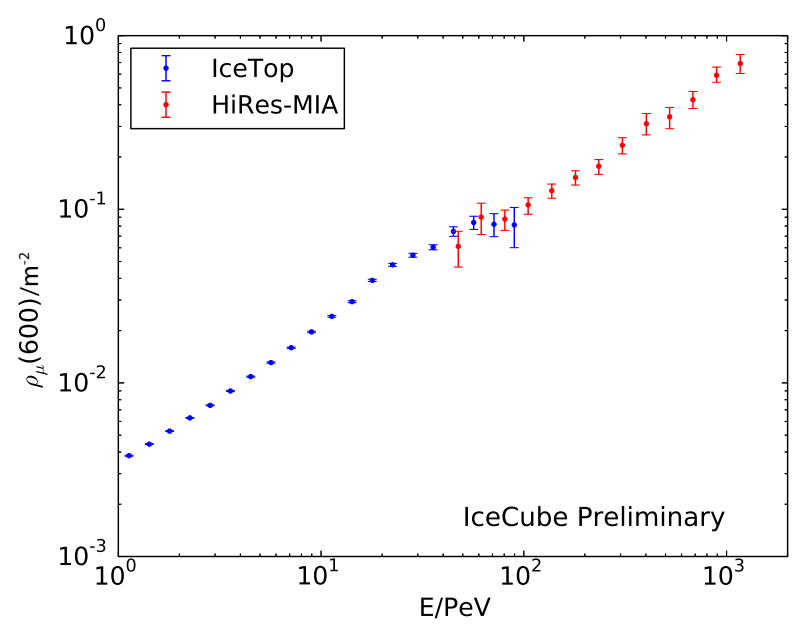

Figure 9. The energy dependence of the muon density $\rho_{\mu}(600)$ for vertical showers. Also shown is the result from the HiResMIA collaboration [2].

\section{Results and discussion}

The resulting muon density at $600 \mathrm{~m}$ from the shower axis is displayed in Fig. 9 for the case of vertical events. Here one can see that the muon density is in the same order of magnitude as the Hires-MIA experiment [2]. One remarkable result is that the dependence on energy agrees very well with the Akeno air shower array result [10,11], which reported a power law dependence with an index of 0.83 .

The interpretation of any apparent differences in the absolute scale in Fig. 9 requires some care. We must remember that the MIA array was not located at the same atmospheric depth as IceTop (the depths are 860 and $680 \mathrm{~g} / \mathrm{cm}^{2}$ respectively), which means that the densities need to be corrected to account for attenuation in the atmosphere. Additionally, the detection thresholds are slightly different. However, it is very encouraging that the apparent offset in Fig. 9 is on the order of the expected attenuation.

At this moment we do not believe that any deviation from a power law behavior in Fig. 9 should be given much importance. The cause for such deviations can still be due to limitations of our method or the choice of lateral distance cut. These and other systematic effects are currently under study.

A more detailed comparison with the expectation from simulations will remain for future contributions, after we have taken into consideration all relevant systematic effects. In this contribution we note that the statistical errors in the present measurement are significantly smaller than any other previous measurement. We expect to make a statement on the absolute scale of the muon density at large lateral distances in the near future.

We are considering improvements to the current analysis. The main limitation at energies larger than $\sim 30 \mathrm{PeV}$ arises from our selection of signals beyond a given lateral distance cut, together with the finite size of the array. These two constraints limit the lever arm in the lateral fit. One of the possibilities we are considering is 
the extension of this analysis to include air showers that go through the detector deep in the ice but not through IceTop, which would increase our lateral distance range. We could also derive improvements from changes in our lateral distance cut.

An obvious application of this measurement will be the improvement of our event-by-event reconstruction to include a muon LDF together with an EM LDF, which we expect will increase our sensitivity to the mass of the primary. This method will provide a measure of primary composition that is independent of the current approach of measuring the high-energy muon bundles deep in the ice $\left(E_{\mu} \gtrsim 300 \mathrm{GeV}\right)$. We expect that a comparison of these two independent measures can help in identifying systematic effects arising from different hadronic models used in Monte Carlo simulations of air showers.

\section{References}

[1] K.H. Kampert, M. Unger, Astropart.Phys. 35, 660 (2012)

[2] Abu-Zayyad, others [HiRes-MIA Collaboration, Phys. Rev. Lett. 84, 4276 (2000)
[3] A. Aab et al. (Pierre Auger Collaboration), Phys. Rev. D (2014)

[4] R. Abbasi et al. (IceCube Collaboration), Nucl. Instrum. Meth. A700, 188 (2013)

[5] R. Abbasi et al. (IceCube Collaboration), Astropart. Phys. 44, 40 (2013)

[6] B. Ruzybayev et al. (IceCube Collaboration), Cosmic ray composition and energy spectrum between $2.5 \mathrm{PeV}$ and $1 \mathrm{EeV}$, in Proc. of the 33rd ICRC \#246, Rio de Janeiro, Brasil (2013), arXiv: 1309.7006

[7] T Feusels et al. (IceCube Collaboration), Measurement of the cosmic ray energy spectrum with IceTop 73, in Proc. of the 33rd ICRC \#861, Rio de Janeiro, Brasil (2013), arXiv:1309.7006

[8] S. Agostinelli et al. Nuclear Instruments and Methods in Physics Research A 506, 250-303 (2003)

[9] K. Greisen, Annual Review of Nuclear Science 10, $63(1960)$

[10] M. Nagano et al., Journal of Physics G: Nuclear Physics 10, 1295 (1984)

[11] N. Hayashida et al., Journal of Physics G: Nuclear and Particle Physics 21, 1101 (1995) 